07

\title{
Аномальное поведение боковой $C-V$-характеристики МНОП-транзистора со встроенным локальным зарядом в нитридном слое
}

\author{
(C) 3.А. Атамуратова, ${ }^{1}$ А. Юсупов, ${ }^{2}$ Б.О. Халикбердиев, ${ }^{1}$ А.Э. Атамуратов ${ }^{1}$ \\ ${ }^{1}$ Ургенческий государственный университет, \\ 220100 Ургенч, Узбекистан \\ 2 Ташкентский университет информационных технологий им. Мухаммада Аль Хорезми, \\ 100200 Ташкент, Узбекистан \\ e-mail: atabek.atamuratov@yahoo.com
}

Поступило в Редакцию 16 августа 2018 г.

В окончательной редакции 2 ноября 2018 г.

Принято к публикации 14 декабря 2018 г.

\begin{abstract}
Выполнено моделирование $C-V$-зависимости для бокового перехода исток-подложка транзистора металл-нитрид-оксид-полупроводник. Встраивание локального ловушечного заряда в нитридный слой приводит к аномальному скачку или спаду емкости перехода при определенных напряжениях на переходе, зависящих от концентрации легирующей примеси в подложке. Такое изменение емкости связано с перераспределением носителей заряда в приповерхностной области подложки, вызванным встраиванием локального заряда. Данная особенность поведения вольт-фарадной характеристики может быть использована для детектирования локального заряда, встроенного в диэлектрический слой полевого транзистора.
\end{abstract}

DOI: 10.21883/JTF.2019.07.47801.319-18

В полевых транзисторах субмикрометровых размеров могут иметь место деградационные эффекты - такие, как эффекты горячих носителей, короткоканальные эффекты, протекание токов утечки, - приводящие к изменениям основных параметров транзисторов. Носители тока, достигшие скорости насыщения в канале, имеют энергию достаточную, чтобы преодолеть оксидный барьер и захватываться на ловушках в диэлектрическом слое или на границе раздела канал-оксид, а также для генерации новых дефектов на границе или в оксидном слое. Это может привести к ухудшению характеристик транзистора: к нестабильности, связанной с неравномерным распределением ловушек, деградации порогового напряжения при обратном смещении затвор-исток/сток, проявляющейся при высоких температурах (NBTI-эффект) [1,2]. Масштаб эффектов, в частности, связан с латеральным распределением концентрации оксидных и граничных дефектов вдоль границы [3].

Кроме этого, наличие конкретного латерального распределения встроенного оксидного заряда, в частности его локализация в ограниченной области, является важной деталью при разработке энергонезависимых элементов памяти, функционирование которых основано на встраивании заряда $[4,5]$. Поэтому задача определения латерального распределения оксидного и/или граничного заряда является одной из важных при изучении характеристик субмикронных полевых транзисторов.

Локальный заряд, встроенный в диэлектрический слой полевого транзистора, в основном идентифицируется с использованием различных видов сканирующей микроскопии [6-8] или метода накачки заряда [5,9]. Сканирующая микроскопия отличается высокой разрешающей способностью, однако при этом она имеет низкую скорость сканирования. Кроме этого, почти все сканирующие микроскопы содержат очень дорогостоящие электромеханические компоненты, обеспечивающие перемещение иглы или образца, и сложные оптические системы (лазер, фотодетектор) для регистрации.

В работе [10] исследовалась возможность регистрации локального заряда, встроенного в нитридном слое транзистора металл-нитрид-оксид-полупроводник (МНОП), измерением вольтфарадной $(C-V)$ характеристики бокового перехода исток-база (сток-база) с использованием пилообразного напряжения, приложенного к этому переходу, без использования зондирующего электрода (иглы). Это позволяет достичь существенной скорости измерения. Но в [10] не были проанализированы особенности („аномалии“) поведения $C-V$-характеристики бокового перехода при встраивании локального заряда и не была рассмотрена трансформация этой характеристики при изменении уровня легирования подложки.

В настоящей работе на основе $2 D$-моделирования изучается аномальное поведение $C-V$-характеристики перехода исток-подложка МНОП-транзистора с локальным зарядом, встроенным в нитридный слой, при концентрациях легирующей примеси в подложке $N_{s u b}$ в пределах между $10^{15} \mathrm{~cm}^{-3}$ и $10^{17} \mathrm{~cm}^{-3}$. Моделирование проводилось с использованием коммерческой программы TCAD Sentaurus. Линейные размеры рабочей области транзистора имеют субмикрометровые масштабы, поэтому применялась диффузионно-дрейфовая модель подвижности, в которой учитывались рекомбинация Шокли-Рида-Холла, насыщение подвижности при высоких полях и влияние нормальной составляющей 


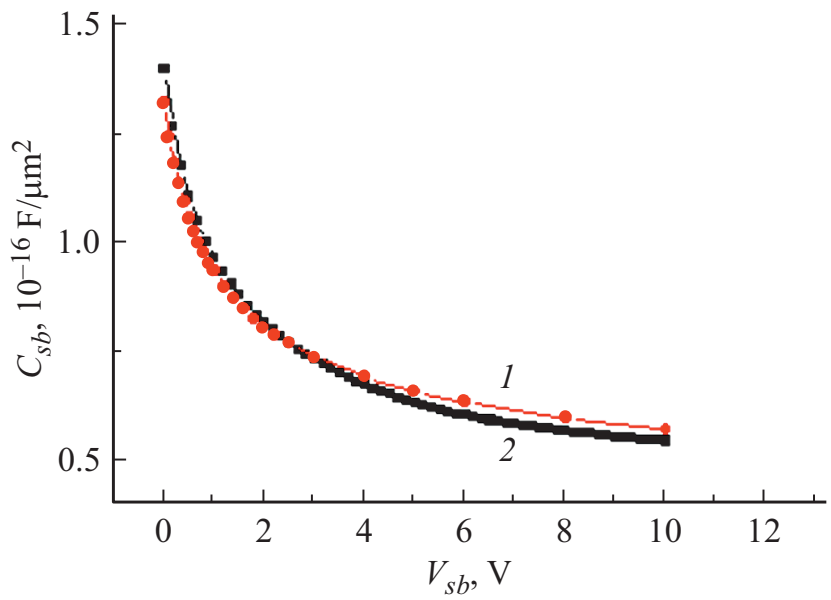

Рис. 1. $C-V$-характеристика для перехода исток-подложка, нормированная к площади перехода: 1 - экспериментальная $C-V$-зависимость полевого транзистора, полученная в работе [11], $2-C-V$-зависимость, полученная моделированием.

поля. Для определения вольт-фарадной характеристики использовался метод слабого переменного сигнала при частоте $1 \mathrm{MHz}$. Модель калибровалась по результатам измерения $C-V$-характеристики перехода исток-подложка без встроенного заряда, приведенной в работе [11] (рис. 1). Калиброванная модель затем использовалась для моделирования вольт-фарадной характеристики перехода исток-подложка МНОП-транзистора с длиной поликремниевого затвора $800 \mathrm{~nm}$, толщинами слоя $\mathrm{SiO}_{2}$ и нитридного слоя $\mathrm{Si}_{3} \mathrm{~N}_{4}$, равными 3 и $50 \mathrm{~nm}$ соответственно. Истоковая и стоковая $n$-области были легированы до концентрации $10^{20} \mathrm{~cm}^{-3}$ (гауссов профиль), а уровень легирования кремниевой $p$-подложки составлял $10^{16} \mathrm{~cm}^{-3}$. Локальный заряд моделировался однородно заряженной областью с ограниченными линейными размерами вдоль канала. Рассматривался заряд длиной $80 \mathrm{~nm}$, который может быть локализован инжекцией в нитридный слой с помощью зонда при приложении соответствующего напряжения [12,13]. Плотность встроенного заряда этой области выбиралась равной $8 \cdot 10^{18} \mathrm{~cm}^{-3}$. Такая объемная плотность соответствует толщине нитридного слоя в $50 \mathrm{~nm}$ при поверхностной плотности $4 \cdot 10^{13} \mathrm{~cm}^{-2}$, наблюдаемой для нитрида $[12,13]$.

Результаты моделирования показывают, что встраивание локального заряда приводит к аномальному скачкообразному изменению $C-V$-характеристики перехода исток-подложка, начиная с определенного напряжения смещения $V_{s b}=V_{\text {jump }}$. Такое поведение емкости может быть использовано для регистрации локального ловушечного заряда в диэлектрических слоях МДП-транзисторов. Характер скачкообразного изменения зависит от уровня легирования подложки. При концентрациях легирования $10^{16} \mathrm{~cm}^{-3}$ и меньше наблюдается скачкообразное увеличение (рис. 2, $a$ ), а при $N_{\text {sub }}>10^{16} \mathrm{~cm}^{-3}-$ скачкообразный спад емкости (рис. 2,b). Для ясной визуализации скачка емкости и идентификации $V_{\text {jump }}$ удобным является определение производной от изменения емкости по напряжению $d(\Delta C) / d V$ из вольт-емкостных характеристик (рис. 3). Очевидно, скачок емкости связан с перераспределением концентрации носителей около поверхности канала, которое вызвано присутствием локального встроенного в нитрид заряда и приводит (при определенных значениях напряжения смещения) к неадекватному изменению величины накапливаемого около перехода заряда при изменении смещения, прилагаемого к переходу исток-подложка.

На рис. 4 и 5 показаны типичные распределения неосновных носителей, электронов, при различных напряжениях смещения на переходе, на глубине $200 \mathrm{~nm}$ от поверхности как при отсутствии, так и после встраивания локального заряда длиной $d=80 \mathrm{~nm}$ на расстоянии $L=150 \mathrm{~nm}$ от истока.

В случае $N_{s u b} \leq 10^{16} \mathrm{~cm}^{-3}$ (рис. 4) встраивание локального заряда в нитридном слое приводит к появлению локального максимума распределения концентрации неосновных носителей в подложке (рис. 4, группа
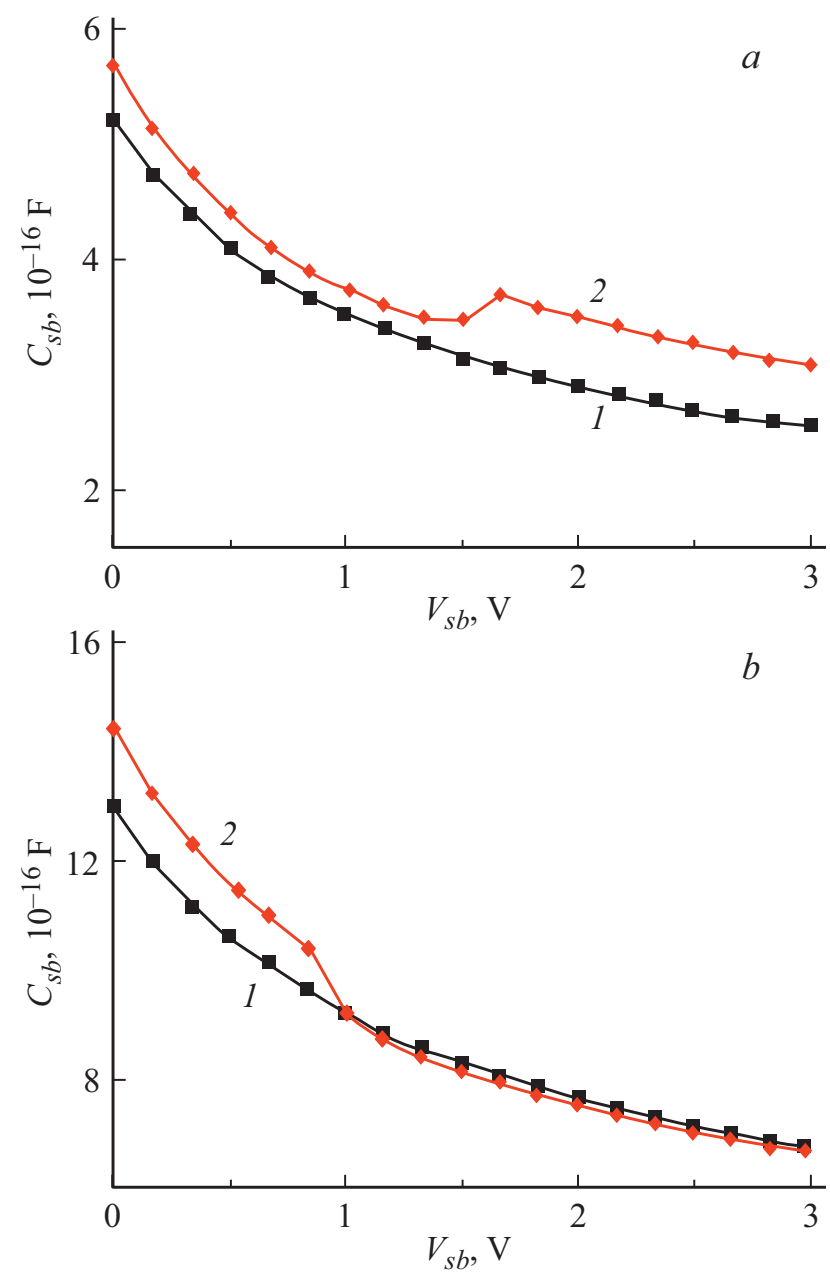

Рис. 2. Зависимость емкости на переходе исток-подложка от напряжения для МНОП-транзистора без встроенного заряда (1) и со встроенным в нитридном слое локальным зарядом на расстоянии $L=150 \mathrm{~nm}$ от истока (2), при уровнях легирования в подложке $N_{s u b}=10^{16} \mathrm{~cm}^{-3}(a)$ и $10^{17} \mathrm{~cm}^{-3}(b)$. 

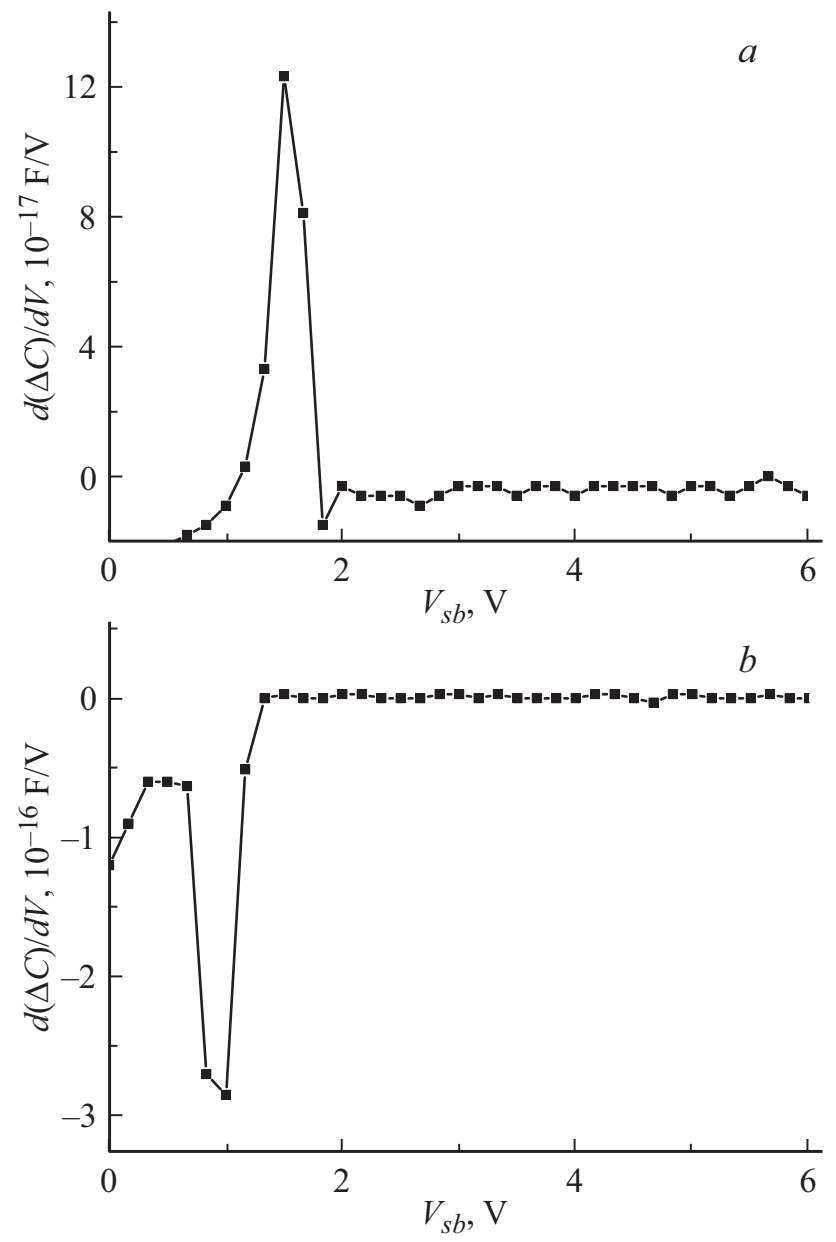

Pис. 3. Производная от изменения емкости по напряжению, $d(\Delta C) / d V$, определенная из вольт-емкостных характеристик до и после встраивания локального заряда в случае $N_{\text {sub }}=10^{16} \mathrm{~cm}^{-3}\left(\right.$ a) и $10^{17} \mathrm{~cm}^{-3}(b)$.

кривых 1), в отличие от случая отсутствия локального встроенного заряда (рис. 4, группа кривых 2). Кривые распределения в каждой группе на рисунке соответствуют различным напряжениям $V_{s b}$ на переходе исток-подложка. Уровень легирования подложки на рис. 4 соответствует $N_{s u b}=10^{16} \mathrm{~cm}^{-3}$. Из рисунка видно, что амплитуда локального максимума зависит от величины напряжения смещения, прилагаемого к переходу исток-подложка (база). С увеличением напряжения смещения амплитуда распределения и полуширина максимума уменьшаются, сама кривая распределения сужается. Однако при рассматриваемой локализации встроенного заряда, начиная с напряжений $V_{s b}=1.7 \mathrm{~V}$, c увеличением напряжения левое крыло локального максимума (группа кривых 3) почти не сдвигается. Это приводит к тому, что амплитуда максимума и полуширина максимума почти не уменьшаются при указанных напряжениях. То есть на расстояниях от центра, начиная от $x=-0.36 \mu \mathrm{m}$, с увеличением напряжения смещения концентрация электронов (неосновных носителей) перестает уменьшаться. Такое поведение распределения, очевидно, и вызывает аномальный скачкообразный рост емкости (рис. 2,a), вместо нормального уменьшения при напряжениях, начиная с $V_{s b}=1.7 \mathrm{~V}$ для данной локализации заряда.

При $N_{s u b}>10^{16} \mathrm{~cm}^{-3}$ изменение распределения неосновных носителей при встраивании локального заряда имеет другой характер, по сравнению с предыдущим случаем $N_{\text {sub }} \leq 10^{16} \mathrm{~cm}^{-3}$. Для $N_{s u b}=1 \cdot 10^{17} \mathrm{~cm}^{-3}$ на рис. 5 показано типичное распределение неосновных носителей на глубине $200 \mathrm{~nm}$ от поверхности подложки при напряжении смещения на переходе исток-подложка меньшем (рис. 5, $b$, кривые 1,2), равном (кривая 3) и большем (кривые 4,5 ) напряжения резкого спада $V_{j u m p}$, равного $1 \mathrm{~V}$ для данного случая. Как видно из рисунка, при равномерном увеличении напряжения смещения с шагом $0.4 \mathrm{~V}$ область объемного заряда увеличивается, ее граница неравномерно сдвигается к центру канала. При приближении напряжения смещения к $V_{\text {juтр }}=1 \mathrm{~V}$ (переход от $V_{s b}=0.6 \mathrm{~V}$ к $V_{s b}=1.0 \mathrm{~V}$ ) сдвиг границы и увеличение ширины области объемного заряда выражены существенно сильнее по сравнению с предыдущим наращиванием (переход от $V_{s b}=0.2 \mathrm{~V}$ к $\left.V_{s b}=0.6 \mathrm{~V}\right)$, что, видимо, и отражается резким спадом емкости около этого напряжения.

Таким образом, результаты моделирования показывают, что при встраивании локального заряда в нитридный слой МНОП-транзистора наблюдается аномальный скачок (резкий рост при $N_{s u b} \leq 10^{16} \mathrm{~cm}^{-3}$ или резкий спад при $N_{s u b}>10^{16} \mathrm{~cm}^{-3}$ ) емкости бокового перехода исток-подложка, начиная с определенных напряжений

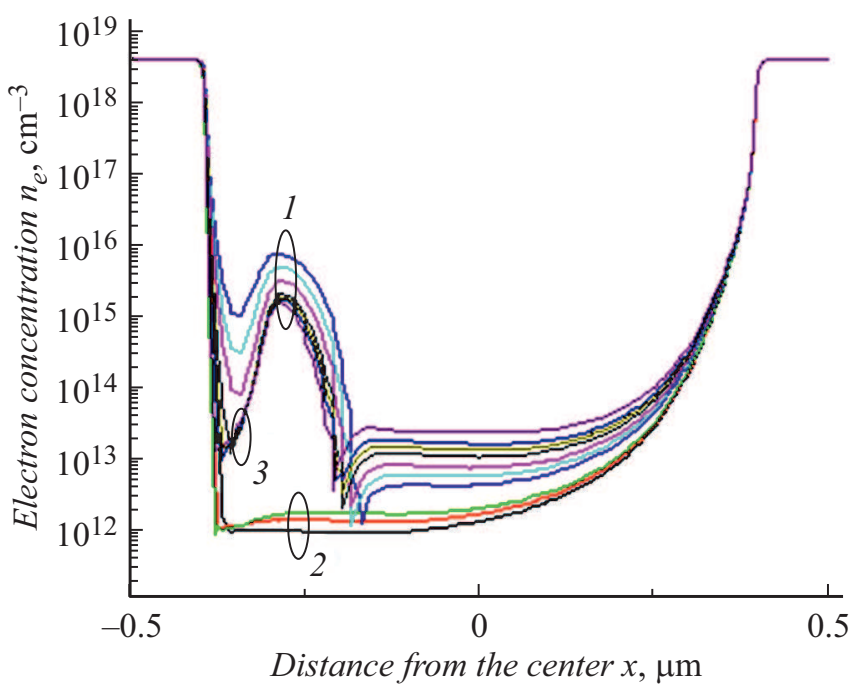

Рис. 4. Распределение концентрации электронов вдоль канала на глубине $200 \mathrm{~nm}$ от поверхности при уровне легирования подложки $N_{\text {sub }}=10^{16} \mathrm{~cm}^{-3}$ в случае встраивания локального заряда на расстоянии $L=150 \mathrm{~nm}$ от истока и при напряжениях $V_{s b}=1,1.2,1.4,1.7,1.9,2.1$ и $2.5 \mathrm{~V}$, где $1 \mathrm{~V}$ соответствует кривой с максимальной амплитудой и $2.5 \mathrm{~V}$ - кривой с минимальной амплитудой (группа кривых 1). Группа кривых 2 соответствует случаю отсутствия локального заряда и напряжениям $V_{s b}=1,1.4$ и $1.7 \mathrm{~V}$. „Слипшаяся“ группа кривых 3 соответствует напряжениям $V_{s b}=1.7,1.9,2.1$ и $2.5 \mathrm{~V}$. 

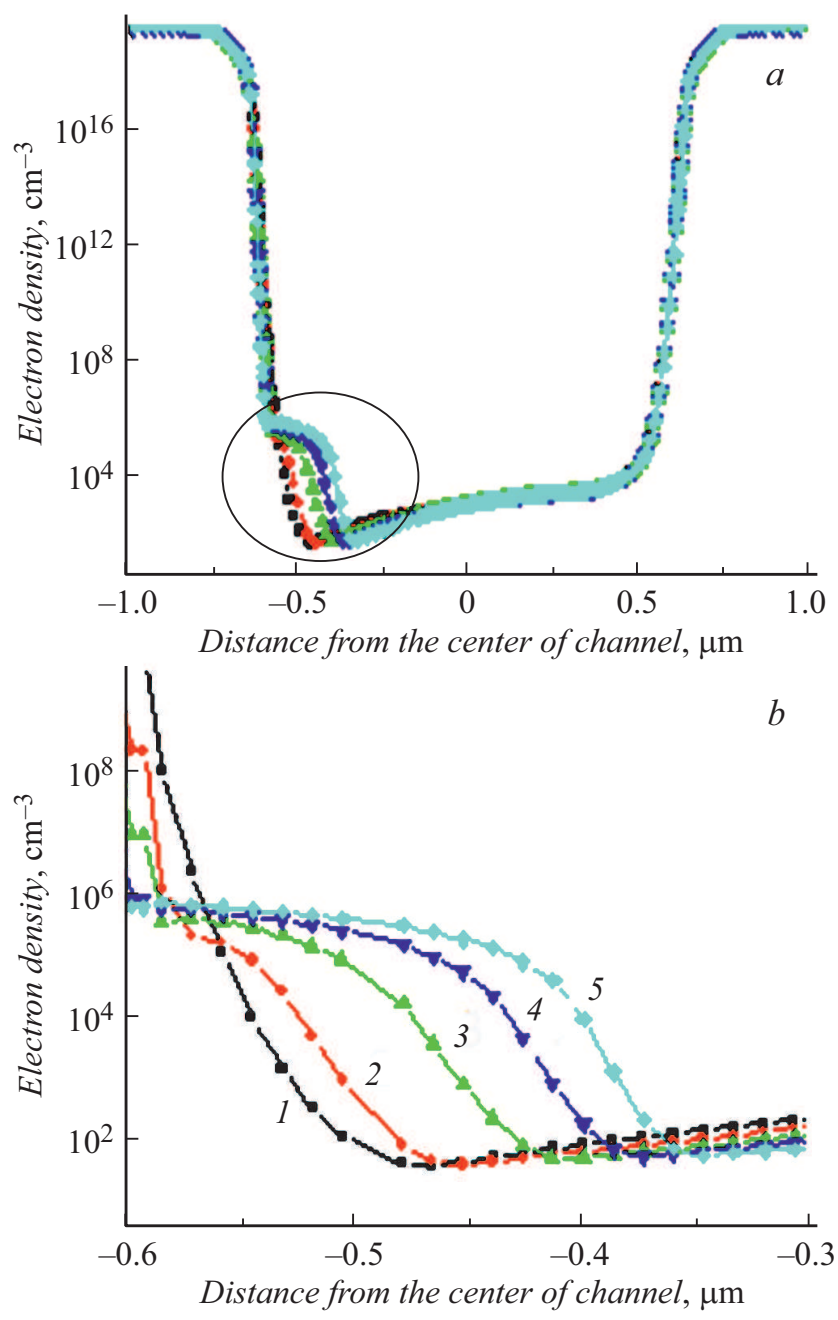

Рис. 5. $a-$ распределение неосновных носителей (электронов) на глубине $200 \mathrm{~nm}$ от поверхности подложки при различных напряжениях смещения $V_{s b}$ на переходе исток-подложка при уровне легирования подложки $N_{s u b}=10^{17} \mathrm{~cm}^{-3} ; b-$ часть рисунка $(a)$, выделенная кругом в увеличенном масштабе; кривые соответствуют различным напряжениям смещения: $1-V_{s b}=0.2,2-0.6,3-1.0,4-1.4,5-1.8 \mathrm{~V}$.

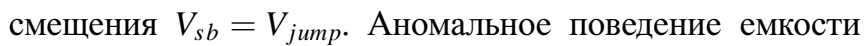
наблюдается в виде пиков на зависимости производной от изменения емкости после встраивания локального заряда по напряжению. Это связано с перераспределением концентрации носителей около поверхности канала, которое вызвано локальным встроенным в нитриде зарядом. Наблюдаемое поведение емкости может быть использовано для определения наличия локального заряда, встроенного в диэлектрик полевого транзистора.

\section{Финансирование работы}

Авторы благодарны Министерству инновационного развития республики Узбекистан за финансовую поддержку исследования, посредством выделения гранта OT- $\Phi 2-67$.

\section{Список литературы}

[1] Kaczer B., Franco J., Weckx P., Roussel Ph.J., Putcha V., Bury E., Simicic M., Chasin A., Linten D., Parvais B., Catthoor F., Rzepa G., Waltl M., Grasser T. // Microelectron. Reliab. 2018. Vol. 81. P. 186-194.

[2] Fleetwood D.M. // Microelectron. Reliab. 2018. Vol. 80. P. 266-277.

[3] Heesoon C., Somyeong S., Jaehoon C., Sunae S. // Curr. Appl. Phys. 2015. Vol. 15. N 11. P. 1412-1416.

[4] Eitan B., Pavan P., Bloom I., Aloni E., Frommer A., Finzi D. // IEEE Electr. Device Lett. 2000. Vol. 21. N 11. P. 543-545.

[5] Rosmeulen M., Breuil L., Lorenzini M., Haspeslagh L., Van Houdt J., De Meyer K. // Solid-State Electron. 2004. Vol. 48. N 9. P. $1525-1530$.

[6] Barrett R.C., Quate C.F. // J. Appl. Phys. 1991. Vol. 70. P. $2725-2733$.

[7] Girard P. // Nanotechnology. 2001. Vol. 12. P. 485-490.

[8] Shin H., Hong S., Moon J., Up Jeon J. // Ultramicroscopy. 2002. Vol. 91. N 1-4. P. 103-110.

[9] Groeseneken G., Maes H.E. // Microelectron. Reliab. 1998. Vol. 38. P. 1379-1389.

[10] Atamuratov A.E., Aminov U.A., Atamuratova Z.A., Halilloev M., Abdikarimov A.A., Matyakubov H. // Nanosystems: Physics, Chemistry, Mathematics. 2015. Vol. 6. N 6. P. 837-842.

[11] Атамуратов А.Е., Матрасулов Д.У., Хабибуллаев П.К. // Докл. РАН. 2007. Вып. 414. № 6. С. 761-764. [Atamuratov A.E., Matrasulov D.U., Khabibullaev P.K. // Doklady Physics. 2007. Vol. 52. N 6. P. 322-325.]

[12] Yatsuda Y., Nabetani S., Uchida K., Minami S.-I., Terasawa M., Hagiwara T., Katto H., Yasni T. // IEEE Tr. Electron. Dev. 1985. Vol. 32. P. 224-234.

[13] Akamine S., Barrett R.C., Quate C.F. // Appl. Phys. Lett. 1990. Vol. 57. P. 316-321. 